\title{
LA VILLA DE LOS PAPIROS DE HERCULANO EN EL SIGLO XXI: ACTUALIZACIÓN CIEN- TÍFICA Y ESTADO DE LA CUESTIÓN (2000- 2016) ${ }^{1}$
}

\author{
Profa. Dra. María Paz López Martínez ${ }^{2}$ \\ Prof. Dr. Andrés Martín Sabater Beltrá ${ }^{3}$
}

\begin{abstract}
Resumen: Nuestro propósito es valorar qué aporta al Humanismo el legado que, oculto durante veinte siglos, apareció en uno de los recintos arqueológicos más interesantes que se conocen en la actualidad, la Villa de los Papiros de Herculano, sepultada por la lava del Vesubio en el año 79 d.C. A partir de 1750, empezó a ser excavada, lo que permitió que se descubriera una lujosa mansión que, además de otras piezas de gran valor, contenía la única biblioteca que ha llegado hasta nosotros desde la Antigüedad. Estos fondos están constituidos por casi dos mil rollos de papiro escritos en griego y atribuidos a un autor oriundo de Siria llamado Filodemo de Gádara. Hace XXI siglos, congregó a los simpatizantes de una escuela cuyo objetivo fundamental era búsqueda de la felicidad. Estamos convencidos de que el mensaje de alegría de vivir, serenidad y tolerancia de Epicuro está más vivo que nunca. Nuestro fin es poner al alcance de un buen número de personas, no sólo de los interesados en el pensamiento, la literatura, la historia y la arqueología helenística e imperial, sino de cualquier persona culta con inquietudes humanísticas todo este rico y complejo legado. Palabras clave: Herculano, Villa dei Papyri, Filodemo, actualización bibliográfica, siglo XXI
\end{abstract}

1 Este artículo supone la reelaboración y actualización de la memoria que, como investigadora principal, presenté (María Paz López Martínez) para solicitar un proyecto de investigación en la convocatoria de Retos de la Sociedad abierta por el Ministerio de Economía, Industria y Competitividad del Gobierno de España durante el año 2015 y que llevó por título Los papiros de Herculano: Textos y contextos históricos y arqueológicos. Su importancia en la bistoria del pensamiento antiguo y moderno y que no se me concedió. A los colegas que entonces aceptaron mi invitación para formar parte del equipo de investigación, les quiero dar las gracias públicamente por su valiosa colaboración durante la redacción de dicha memoria: Dr. Gianluca del Mastro, Università degli Studi di Napoli Federico II; Dr. Juergen Hammerstaedt, Universität zu Köln; Holger Essler, Universität Würzburg; Dr. George Karamanolis, Universität Wien; Dr. Gustavo Castel de Lucas, Universidad Complutense; Dr. Javier Fresnillo Núñez, Universidad de Alicante y Dr. José Uroz Sáez, Universidad de Alicante. También, quiero expresar mi agradecimiento, de manera muy especial, al Dr. Javier Campos Daroca de la Universidad de Almería, a la Dra. Montserrat Jufresa Muñoz de la Universidad de Barcelona y al propio Dr. Andrés Martín Sabater Beltrán, que se prestaron también a colaborar en el proyecto. Por último, a la Dra. Carmen Morenilla Talens de la Universidad de Valencia.

2 Professora Titular da Universidad de Alicante (Espanha).E-mail: maripaz.lopez@ua.es.

3 Doutor em Prehistoria, Arqueología, Historia Antigua, Filología Griega y Filología Latina pela Universidad de Alicante (Espanha).E-mail: andpapyrifer@hotmail.es. 


\section{INTRODUCCIóN}

El propósito de estas páginas es presentar una revisión bibliográfica de una parte importante - el informe tiene pretensión de exhaustividad- de los estudios que se han publicado en los últimos años sobre los materiales aparecidos en la Villa de los Papiros de la ciudad de Herculano, concretamente, durante los primeros años de este siglo XXI recientemente inaugurado. Fundamentalmente, se trata de facilitar un punto de partida, de una invitación a participar en una reflexión más profunda sobre el interés científico que supone el conocimiento de esta rica rama de la investigación humanística de cara a ámbitos profesionales y académicos que habitualmente se mantienen alejados del estricto marco de los Estudios Clásicos, la Filosofía, la Arqueología o la Historia Antigua. Nos referimos a disciplinas como Psicología, Medicina o Sociología, por citar sólo algunas de aquéllas que podrían beneficiarse del contacto con este atractivo y complejo patrimonio material e inmaterial que ha estado oculto durante más de veinte siglos.

\section{ESTADO dE LA CUESTIÓN}

El núcleo central es uno de los recintos arqueológicos más interesantes que se conocen en la actualidad, la Villa de los Papiros de Herculano. Se trata de una casa romana sepultada por la lava del Vesubio en el año 79 d.C. Dicha vivienda empezó a ser excavada a partir de 1750, cuando se descubrió una lujosa mansión que, además de otras piezas de gran valor, contenía la única biblioteca que ha llegado hasta nosotros desde la Antigüedad. Los fondos de esta biblioteca están constituidos por casi dos mil rollos de papiro escritos en griego y atribuidos a un autor oriundo de Jordania llamado Filodemo de Gádara.

Nuestro punto de partida son los textos. Éstos hacen referencia a temas diversos entre los que destacan los tratados de filosofía, historia de la filosofía, teología, ética y estética, entre otros. Dadas las condiciones en las que se encuentran los papiros, desde su descubrimiento hasta hoy se han dedicado muchos esfuerzos a los complejos procesos de apertura, lectura y edición de los rollos carbonizados y debemos subrayar que, aunque en un principio se utilizaron metodologías bastante agresivas para poder acceder a ellos, en las últimas décadas, la lectura y edición de los manuscritos se ha beneficiado de avances tecnológicos que, por otro lado, obligan a revisar la mayoría de las ediciones anteriores al siglo XXI. 
Durante los últimos años, se ha estudiado también en profundidad el propio enclave en el que se han encontrado los papiros, en sus aspectos arqueológicos e históricos. Lo mismo ha sucedido con respecto a la historia de su descubrimiento y al impacto que causó en la intelectualidad europea de la época.

Añadiremos, además, que Herculano no fue el único foco de difusión de la escuela epicúrea. Además de la Campania, encontramos testimonios interesantes en otros puntos geográficos de la periferia, como por ejemplo, en la ciudad de Enoanda situada en la actual Turquía.

A las labores de edición, traducción y comentario de los textos, siguen los estudios e interpretaciones del singular enclave arqueológico en el que han encontrado los papiros. Sin temor a exagerar, podemos decir que éste constituye, en la actualidad, uno de los conjuntos patrimoniales y de los destinos turísticos más ricos y atractivos de Europa. Por otro lado, los hallazgos de la zona vesubiana han sido capitales para el desarrollo del arte neoclásico gracias al desarrollo del gusto herculanense, convertido en un fenómeno europeo del cual se pueden encontrar huellas en la más variada tipología de objetos (mobiliario, vajillas, enseres domésticos...).

Herculano congregó, hace veintiun siglos, a los simpatizantes de una escuela -mujeres, hombres, esclavos, libres, ciudadanos o extranjeros, todo el mundo estaba admitido- cuyo objetivo fundamental era búsqueda de la felicidad.

Unas de las riquezas fundamentales en este entorno son los textos epicúreos que aparecieron en la famosa Villa dei Papiri y de su biblioteca, en su mayoría de Filodemo de Gádara, protegido del patronus de la villa, Lucio Calpurnio Pisón, un conspicuo miembro de la nobilitas romana, cónsul en el 58 a.C. y suegro de César.

Fruto de las primeras excavaciones arqueológicas en la región vesubiana fueron las reproducciones de las piezas arqueológicas que Carlos (VII de Nápoles y más tarde Carlos III, rey de España) encargó a los más prestigiosos artistas y estudiosos del momento. Dicho encargo dio como fruto una colección de ocho tomos titulada Le Antichità di Ercolano Esposte editados en la Regia Stamperia de Nápoles en 1757-1796. Estos volúmenes, junto con algunos otros conservados en la Real Academia de Bellas Artes de San Carlos de Valencia, forman parte del catálogo de obras maestras del arte tipográfico del siglo XVIII y, al ser utilizados en la Real Academia para la formación de sus alumnos, contribuyeron a la formación del gusto herculanense, como se conoce a una de las últimas manifestaciones del arte barroco y rococó. De modo que 
podemos afirmar que estas espléndidas ilustraciones contribuyeron de manera notable a la formación del gusto neoclásico en Europa desde finales del siglo XVIII y durante todo el siglo XIX.

Asimismo, las revistas y publicaciones científicas europeas, con tal de mantener informados a sus lectores, recopilaron artículos de especialistas y extractos de cartas que los encargados de los papiros enviaban al rey o a sus familiares y amigos con comentarios acerca de estos documentos. Otra alternativa de la que disponían los lectores más curiosos era recurrir a los libros de viaje redactados por aquéllos que habían disfrutado el privilegio de visitar las excavaciones y el citado museo. Fue proverbial el espíritu viajero de franceses, británicos y alemanes. Concretamente, fueron las cartas de Winckelmann las que descubrieron los papiros ante el público europeo. En España, cultivaron este género prestigiosos intelectuales y escritores de la talla de Francisco Pérez Báyer, Nicolás de Azara, Esteban de Arteaga, Pedro Montengón, Leandro Fernández de Moratín, Pedro Antonio de Alarcón y Juan Andrés y Morell.

Desde mediados del siglo XVIII hasta la actualidad se ha llevado a cabo una labor prácticamente ininterrumpida de apertura, catalogación, conservación y traducción y, aunque en el mundo académico las dificultades derivadas de la condición material de los rollos y el contenido filosóficoepicúreo de los primeros ejemplares hicieron decaer el interés por los mismos durante un largo periodo de tiempo, el avance de la metodología y la técnica experimentado en las tres últimas décadas ha producido una revitalización de estos documentos como objeto de estudio.

En este contexto hay que destacar el esfuerzo realizado por Marcello Gigante y el CISPE (Centro Internazionale per lo Studio dei Papiri Ercolanesi "Marcello Gigante" [http://www.cispe.org -fecha de la consulta: 1-V-2017-]), cuyas publicaciones - especialmente la colección de textos La Scuola di Epicuro y la revista Cronache Ercolanesi- han dado fe de la importancia arqueológica, filológica e histórica de los papiros y los hallazgos de Herculano. Precisamente, este año se inaugura la Prima Scuola Estiva di Papirologia Ercolanese [http:// www.cispe.org/prima-scuola-estiva-di-papirologia-ercolanese/ -fecha de la consulta: 1-V-2017-].

$\mathrm{Al}$ mismo tiempo han ido surgiendo agrupaciones como The Friends of Herculaneum Society [http://www.herculaneum.ox.ac.uk -fecha de la consulta: 1-V-2017-], encargada de difundir información sobre los restos procedentes de las excavaciones, y el grupo de investigación TELEPHe (Traduire Ensemble en Langues Européennes les Papyrus d'Herculanum), que incluye varias universidades y centros de investigación - Universidad de Alicante, Universidad 
de Barcelona, Humboldt-Universität zu Berlin, l'Université de Bordeaux 3. University of Cambridge, Universität zu Köln, Università del Salento, Lecce, Università degli Studi Federico II di Napoli, University of Oxford, Université de Paris-Sorbonne (ParisIV), Università degli Studi di Siena, Année Philologique, Universität Würzburg y Université Charles de GaulleLille3-. El objetivo de TELEPHe es la edición y traducción de los textos en diferentes lenguas europeas. Desde el año 2006, sus componentes procuran reunirse cada año, cada dos años o con la mayor frecuencia posible, en una sede diferente (Lille 2006, Barcelona 2007, Cambridge 2008, París 2009, Ginebra 2010, Varsovia 2013, Barcelona 2015) con el fin de poner en común el trabajo desarrollado por los miembros del equipo. La dinámica habitual consiste en la discusión de los pasajes que resultan especialmente problemáticos. El grupo se beneficia de los avances tecnológicos que se están aplicando al estudio de los papiros de Herculano como son las fotografías multiespectrales. En esta línea, destaca también The Philodemus Project [http://classics.ucla.edu/faculty -projects/philodemus-project/ -fecha de la consulta: 1-V-2017-], centrado en la traducción al inglés de la obra estética de Filodemo, al que debemos la autoría de gran parte de los tratados que se conservaban en la mencionada biblioteca.

La mayor parte de los papiros se conservan en la Biblioteca Nacional de Nápoles. Los viejos microscopios han sido substituidos por otros más potentes y por una tecnología más sofisticada que permite trabajar desde un ordenador que no esté en la Officina: las fotografías se escanean, después se digitalizan y se hacen multiespectrales. Por otra parte, Daniel Delattre y Dirk Obbink estudiaron un sistema que permitía conocer la secuencia correcta de los diseños de siglo de los siglos XVIII y XIX y asimismo varios estudiosos han elaborado métodos matemáticos para reunir los fragmentos de los papiros de Herculano, reconstruyendo virtualmente la posición originaria interna del rollo.

La utilización de microscopios avanzados, las microfotografías, el desarrollo de los ordenadores y del método osloense, y la aplicación de la fotografía multiespectral han mejorado las labores de apertura y lectura, planteando la obligación de revisar las ediciones anteriores al siglo XXI. Precisamente en marzo del 2015 se hizo público un avance espectacular en este sentido. Se trata de un nuevo método para leer estos papiros carbonizados mediante el empleo de una tomografía cuya presentación corrió a cargo de Daniel Delattre en Oxford. La prestigiosa revista Nature Communications se hizo eco de este importante descubrimiento [http://www.nature.com/ ncomms/2015/150120/ncomms6895/full/ncomms6895.html - fecha de 
la consulta: 1-V-2017]. En España, la exposición La Villa de los Papiros, inaugurada el día 17 de octubre de 2013 en Madrid y organizada por Casa del lector, disfrutó de gran éxito.

\section{Publicaciones del siglo XXI}

\section{1- Relatos de viajeros}

- ANTONI, Agathe. L'Officina des Papyrus dans la description de Vivant Denon. Cronache Ercolanesi, Nápoles, n. 32, 2002, p. 321-324.

- ANTONI, Agathe. Voyageurs français à la découverte d'Herculanum aux XVIIİ̀me et XIXème siècles. Cronache Ercolanesi, Nápoles, n. 39, 2009, p. 313-330.

- BUONAJUTO, Alessandra. I papiri ercolanesi nelle memorie dei viaggiatori inglesi del Settecento. Cronache Ercolanesi, Nápoles, n. 30, 2000, p. 243-244.

- LONGO AURICCHIO, Francesca. Luna e gamberi. Su una locuzione impiegata da Winckelmann. Cronache Ercolanesi, Nápoles, n. 37, 2007, p. 135-139.

- LÓPEZ MARTÍNEZ, María Paz - SABATER BELTRÁ, Andrés Martín. Los papiros de Herculano en la España de finales del siglo XVIII y comienzos del XIX. Cronache Ercolanesi, Nápoles, n. 41, 2011, p. 261-271.

- MINUTOLI, Diletta - PINTAUDI, Rosario. Angelo Maria Bandini e la sua visita a Portici (31 gennaio 1781). Cronache Ercolanesi, Nápoles, n. 32, 2002, p. 301-303.

- SABATER BELTRÁ, Andrés Martín. Los papiros de Herculano en los relatos de viajeros del siglo XVIII. En: CUTILLAS, Ernesto. Investigar en Humanidades: Actas de las IV Jornadas de Investigación de la Facultad de Filosofía y Letras. San Vicente del Raspeig: Publicaciones de la Universidad de Alicante, 2015, p. 49-55.

\section{2-Excavaciones y descubrimientos}

. CIARDIELLO, Rosaria. Archaelogia ercolanese. Cronache Ercolanesi, Nápoles, n. 31, 2001, p. 139-141.

- CIARDIELLO, Rosaria. Le sculture dalla Villa dei Papiri a Ercolano: nuove metodologie e tecniche diagnostiche. Cronache Ercolanesi, Nápoles, n. 37, 2007, p. 161-169.

- CIARDIELLO, Rosaria. La fortuna delle scoperte ercolanesi e pompeiane e la manifattura di gemme tra il XVII e il XIX secolo. Cronache Ercolanesi, Nápoles, n. 46, 2016, p. 217-224.

- DE SIMONE, Antonio. Rilievo con satiri e ninfa. Testa di Amazzone. En: PAGANO, Mario. Gli antichi ercolanesi: antropologia, società, economia: guida alla mostra. Nápoles: Electa, 2000, p. 22-23.

- DE SIMONE, Antonio - RUFFO, Fabrizio. Ercolano 1996-1998. Lo scavo della Villa dei Papiri. Cronache Ercolanesi, Nápoles, n. 32, 2002, p. 325-344. 
- DE SIMONE, Antonio - RUFFO, Fabrizio. Ercolano e la Villa dei Papiri alla luce dei nuovi scavi. Cronache Ercolanesi, Nápoles, n. 33, 2003, p. 279-311.

- DE SIMONE, Antonio - RUFFO, Fabrizio. I mosaici della Villa dei Papiri ad Ercolano (Na). Il quartiere dell'atrio. En: ANGELLELI, Claudia. Atti del X colloquio dell'Associazione italiana per lo studio e la conservazione del mosaico: Lecce, 18 21 febbraio 2004. Tívoli, 2005, p. 161-182.

- DE SIMONE, Antonio. La villa dei Papiri ad Ercolano. En: CIARDIELLO, Rosaria. La Villa romana. Nápoles: Lorientale, 2007, p. 167-193.

- DEL MASTRO, Gianluca. Un capitolo sulla biblioteca ercolanese. Cronache Ercolanesi, Nápoles, n. 46, 2016, p. 169-181.

- ESPOSITO, Domenico. Un contesto di pitture inedito dalla cosiddetta Palestra di Ercolano. Cronache Ercolanesi, Nápoles, n. 45, 2015, p. 209-225.

- GUIDOBALDI, Maria Paola - ESPOSITO, Domenico. Le nuove ricerche archeologiche nella Villa dei Papiri di Ercolano. Cronache Ercolanesi, Nápoles, n. 39, 2009, p. 331-370.

- JANKO, Richard. The Herculaneum Library: Some Recent Developments. Estudios Clásicos, Madrid, n. 121, 2002, p. 25-41.

- LONGO AURICCHIO, Francesca. La Villa Ercolanese dei Papiri: storia delle scoperte e vita dell'Officina dal Museo di Portici al Palazzo Reale di Napoli. Cronache Ercolanesi, Nápoles, n. 30, 2000, p. 11-20.

- LONGO AURICCHIO, Francesca. Sullo scavo della Villa dei Papiri. Cronache Ercolanesi, Nápoles, n. 38, 2008, p. 319-328.

- MOESCH, Valeria. La Villa dei Papiri. En: GUIDOBALDI, Maria Paola. Ercolano, tre secoli di scoperte: Napoli, Museo Archeologico Nazionale, 16 ottobre 2008-13 aprile 2009. Milán: Electa, 2008, p. 70-79.

- NOTOMISTA, Mario. Lo scavo dell'area sacra di Herculaneum. Storia degli scavi e definizione del culto. Cronache Ercolanesi, Nápoles, n. 46, 2016, p. 183-216.

- PAGANO, Enrica. Caylus mécène du roi. Collectionner les antiquités au XVIII ${ }^{\mathrm{e}}$ siècle. Cronache Ercolanesi, Nápoles, n. 34, 2004, p. 209-211.

. PAGANO, Mario. Gli scavi di Ercolano. Nápoles: Marius, 2003.

- PAGANO, Mario - PRISCIANDARO, Raffaele. Studio sulle provenienze degli oggetti rinvenuti negli scavi boronici del Regno di Napoli: una lettura integrata, coordinata e commentata della documentazione. Castellammare di Stabia (Nápoles): N. Longobardi, 2006.

- QUILICI, Vieri. - LONGOBARDI, Giovanni. Ercolano e la Villa dei Papiri: archeologia, città e paesaggio. Florencia: Alinea, 2007.

- ZARMAKOUPI, Mantha. The Villa of the Papyri at Herculaneum: archaeology, reception, and digital reconstruction. Alemania: Walter de Gruyter GmbH \& Co. KG, Göttingen, 2010. 


\section{3- Los papiros desde una perspectiva general}

- BLANK, David L. - LONGO AURICCHIO, Francesca. An Inventory of the Herculaneum Papyri from Piaggio’s Time . Cronache Ercolanesi, Nápoles, n. 30, 2000, p. 131-147.

- BLANK, David L. - LONGO AURICCHIO, Francesca. Su alcuni inventari antichi dei Papiri Ercolanesi . Cronache Ercolanesi, Nápoles, n. 32, 2002, p. 297-300.

- BLANK, David L. - LONGO AURICCHIO, Francesca. Inventari antichi dei Papiri Ercolanesi. Cronache Ercolanesi, Nápoles, n. 34, 2004, p. 39-152.

- CAMODECA, Giuseppe - DEL MASTRO, Gianluca. I papiri documentari ercolanesi (PHerc. MAN): relazione preliminare. Cronache Ercolanesi, Nápoles, $\mathrm{n}$. 32, 2002, p. 281-296.

- CAPASSO, Mario. Il Catalogo multimediale dei Papiri Ercolanesi. Cronache Ercolanesi, Nápoles, n. 36, 2006, p. 219-226.

- CAPASSO, Mario. I rotoli ercolanesi: da libri a carboni e da carboni a libri. En: PALME, Bernhard. Akten des 23 Internationalen Papyrologenkongresses, Wien, 22-28, Juli 2001. Papyrologica Vindobonensia 1. Viena: Verlag der Österreichischen Akademie der Wissenschaften, p. 73-77.

- CARRELLI, Sergio. Un nuovo punto di vista sulla consistenza della collezione dei papiri ercolanesi. Cronache Ercolanesi, Nápoles, n. 46, 2016, p. 127-136.

- DE GIANNI, Angelica - NAPOLITANO, Stefano. Francesco Casanova disegnatore dei papiri ercolanesi. Cronache Ercolanesi, Nápoles, n. 46, 2016, p. 137 159.

- DEL CORSO, Lucio. Ercolano e l'Egitto: pratiche librarie a confronto. Cronache Ercolanesi, Nápoles, n. 43, 2013, p. 139-160.

- DEL MASTRO, Gianluca. Secondo supplemento al Catalogo dei Papiri Ercolanesi. Cronache Ercolanesi, Nápoles, n. 30, 2000, p. 157-241.

- DEL MASTRO, Gianluca. Reflessioni sui papiri latini ercolanesi. Cronache Ercolanesi, Nápoles, n. 35, 2005, p. 183-194.

- DEL MASTRO, Gianluca. Osservazioni bibliologiche e paleografiche su alcuni papiri ercolanesi. Cronache Ercolanesi, Nápoles, n. 39, 2009, p. 283-299.

- DEL MASTRO, Gianluca. Il Catalogo descrittivo dei Papiri Ercolanesi. Cronache Ercolanesi, Nápoles, n. 40, 2010, p. 215-222.

- DEL MASTRO, Gianluca. Titoli e annotazioni bibliologiche nei papiri greci di ercolano. Centro Internazionale per lo studio dei papiri ercolanesi Marcello Gigante, Quinto Supplemento a Cronache Ercolanesi, Nápoles, 2014.

- DELATTRE, Daniel. Reconstruire virtuellement les livres carbonisés d'Herculanum: premier bilan d'une orientation prometteuse de la recherche. Cronache Ercolanesi, Nápoles, n. 40, 2010, p. 191-214.

- DELATTRE, Daniel. Cronistoria dei papiri ercolanesi conservati a Parigi (18022012). Cronache Ercolanesi, Nápoles, n. 44, 2014, p. 129-144. 
. DORANDI, Tiziano. Theodor Gomperz (1832-1912). En: CAPASSO, Mario. Hermae: Scholars and Scholarship in Papyrology. Pisa: Giardini, 2007, p. 29-43.

- ESSLER, Holger. Bilder von Papyri und Papyri als Bilder. Cronache Ercolanesi, Nápoles, n. 36, 2006, p. 103-143.

- ESSLER, Holger. Rekonstruktion von Papyrusrollen auf mathematischer Grundlage. Cronache Ercolanesi, Nápoles, n. 38, 2008, p. 273-307.

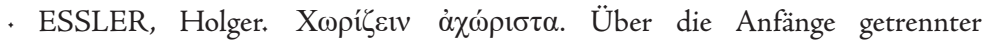
Aufbewahrung der herkulanischen Papyri. Cronache Ercolanesi, Nápoles, n. 40, 2010, p. 173-189.

- INDELLI, Giovanni. Testimonianze su Pericle nei papiri di Filodemo. Cronache Ercolanesi, Nápoles, n. 32, 2002, p. 233-238.

- INDELLI, Giovanni. Osservaioni sul lessico artistico nei testi ercolanesi. Cronache Ercolanesi, Nápoles, n. 38, 2008, p. 179-192.

- INDELLI, Giovanni. Il lessico di Filodemo in alcune opere morali: gli $\alpha \approx \pi \alpha \xi$ $\lambda \varepsilon \gamma o ́ \mu \varepsilon v \alpha$. Cronache Ercolanesi, Nápoles, n. 40, 2010, p. 87-93.

. KNIGHT, Carlo. Canguri e papiri. Cronache Ercolanesi, Nápoles, n. 32, 2002, p. 305-320.

- LEONE, Giuliana - CARRELLI, Sergio. La morfologia dei papiri ercolanesi: risultati e prospettive di ricerca dall'informatizzazione dell'Inventario del 1782 . Cronache Ercolanesi, Nápoles, n. 45, 2015, p. 147-187.

- LONGO AURICCHIO, Francesca - CHIRICO, Maria Luisa. Un nuovo volume di Contributi alla Storia della Officina dei Papiri Ercolanesi. Cronache Ercolanesi, Nápoles, n. 34, 2004, p. 213-222.

- LONGO AURICCHIO, Francesca. Osservazioni su alcune scorze della biblioteca ercolanese. Cronache Ercolanesi, Nápoles, n. 40, 2010, p. 137-154.

- LÓPEZ MARTÍNEZ, María Paz. Aportación de la Villa dei Papiri de Herculano al Humanismo. En: ARRÁEZ LLOBREGAT, José Luis - RAMÓN DÍAZ, Carmen - SIRVENT RAMOS, Ángeles. Del saber a la vida: Ensayos en homenaje al profesor Francisco Ramón Trives. San Vicente del Raspeig: Publicaciones de la Universidad de Alicante, 2009, p. 227-236.

- MOORMAN, Eric M. Fictitious Manuscripts from Herculaneum, Pompeii, and Antiquity. Cronache Ercolanesi, Nápoles, n. 40, 2010, p. 239-249.

- PORTER, James I. Hearing Voices: The Herculaneum papyri and classical scholarship. En: Gardner Coates, Victoria C. - Seydl, Jon L. Antiquity recovered: the legacy of Pompeii and Herculaneum. Los Ángeles: J. Paul Getty Museum, 2007, p. 95-113.

- SIDER, David. The Special Case of Herculaneum. En: BAGNALL, Roger S. The Oxford Handbook of Papyrology. Nueva York: Oxford University Press, 2009, p. 303-319.

- TRAVAGLIONE, Agnese - DEL MASTRO, Gianluca. Sistemazione dei papiri privi di supporto. Cronache Ercolanesi, Nápoles, n. 35, 2005, p. 215-221. 
- TRAVAGLIONE, Agnese. L'Archivio dell'Officina dei Papiri Ercolanesi on line. Cronache Ercolanesi, Nápoles, n. 36, 2006, p. 227-232.

- TRAVAglione, Agnese. Catalogo Descriptivo dei Papiri Ercolanesi. Nápoles: CISPE - Biblioteca Nazionale “Vittorio Emanuele III”, 2008.

4.4- Apertura de los rollos

- CAPASSO, Mario. Contributi alla storia della Officina dei Papiri Ercolanesi. 3. Nápoles: Graus, 2003.

- CAPASSO, Mario. La Papirologia Ercolanese nel decennio francese a Napoli (1806-1815).En: DI ANTONI, Agathe. - ARRIGHETTI, Graziano. - BERTAGNA, M. Isabella. - DELATTRE, Daniel. Miscellanea Papyrologica Herculanensia. Pisa * Roma: Fabrizio Serra Editore, v. I, Biblioteca di Studi Antichi 93, 2010, p. 247-270.

- DEL MASTRO, Gianluca - DELATTRE, Daniel - MOCELLA, Vito. Una nuova tecnologia per la lettura non invasiva dei papiri ercolanesi. Cronache Ercolanesi, Nápoles, n. 45, 2015, p. 227-229.

- KLEVE, Knut - CAPASSO, Mario - DEL MASTRO, Gianluca. Papiri aperti col metodo osloense (1999). Cronache Ercolanesi, Nápoles, n. 30, 2000, p. 247-249.

- PROTO, Fiorenza. Piano piano, se pol romper. Cronache Ercolanesi, Nápoles, n. 37, 2007, p. 141-160.

- SEALES, Brent W. - DELATTRE, Daniel. Virtual Unrolling of Carbonized Herculaneum Scrolls: Research Status (2007-2012). Cronache Ercolanesi, Nápoles, n. 43, 2013, p. 191-208.

\section{5- Contenido de los papiros}

a) Autores sin identificar

- P.Herc, 1067

- Piano, V. Sull'autore del P.Herc. 1067: una nuova lettura della subscriptio", Analecta Papyrologica, Mesina, n. 28, 2016, p. 273-283.

- Bellum Actiacum

- SCAPPATICCIO, Maria C. Il PHerc. 817: spunti paleografici. Cronache Ercolanesi, Nápoles, n. 38, 2008, p. 229-246.

- SCAPPATICCIO, Maria C. Il PHerc. 817: echi virgiliani e <<pseudoaugusteismo>> . Cronache Ercolanesi, Nápoles, n. 40, 2010, p. 99-136.

- Sobre los amigos de Epicuro

- CAMPOS DAROCA, Francisco J. - LÓPEZ MARTÍNEZ, María P. Lettres de femmes selon le PHerc. 176: la correspondance de Batis. En: DI ANTONI, Agathe. - ARRIGHETTI, Graziano. - BERTAGNA, M. Isabella. - DELATTRE, Daniel. Miscellanea Papyrologica Herculanensia. Pisa - Roma: Fabrizio Serra Editore, v. I, Biblioteca di Studi Antichi 93, 2010, p. 21-36. 
- TEPEDINO GUERRA, A. Le lettere private del KHПOC: Metrodoro, i maestri e gli amici epicurei (PHerc. 176 e PHerc. 1418). En: DI ANTONI, Agathe. - ARRIGHETTI, Graziano. - BERTAGNA, M. Isabella. - DELATTRE, Daniel. Miscellanea Papyrologica Herculanensia. Pisa - Roma: Fabrizio Serra Editore, v. I, Biblioteca di Studi Antichi 93, 2010, p. 37-59.

b) Crisipo

- Obras inciertas

- ANTONI, Agathe. Le PHerc. 1384: édition critique. Cronache Ercolanesi, Nápoles, n. 42, 2012, p. 17-92.

- Cuestiones lógicas

- Sobre los elementos de los enunciados

- DEL MASTRO, Gianluca. Il PHerc. 1380: Crisippo, Opera logica. Cronache Ercolanesi, Nápoles, n. 35, 2005, p. 61-70.

\section{c) Demetrio Lacón}

\section{- Obras inciertas}

- DEL MASTRO, Gianluca. Demetrio Lacone e la correzione degli errori nei testi epicurei (PHerc. 1012, coll. XXXIV 3-9 Puglia). Cronache Ercolanesi, Nápoles, n. 34, 2004, p. 205-208.

- PARISI, Antonio. Osservazioni sul lessico del PHerc. 831 (Demetrio Lacone, Opus incertum). Cronache Ercolanesi, Nápoles, n. 42, 2012, p. 111-119.

- PARISI, Antonio. Le forme del sapere astronomico nell'Epicureismo: un saggio di lettura di PHerc. 831, IX-XI Körte. Cronache Ercolanesi, Nápoles, n. 44, 2014, p. 49-64.

- PARISI, Antonio. Errori e sensazioni: per una nuova lettura di PHerc. 831 (Demetrio Lacone, Opus incertum). Cronache Ercolanesi, Nápoles, n. 46, 2016, p. 39-53.

- VERDE, Francesco. Minimi in movimento? Note sulle coll. XLVIII-L Puglia del PHerc. 1012 (Demetrii Laconis Opus incertum). Cronache Ercolanesi, Nápoles, n. 41, 2011, p. 51-64.

- Sobre algunas investigaciones comunes respecto al modo de vida

- ASSANTE, Maria G. Per un riesame del PHerc. 1006 (Demetrio Lacone, Alcune ricerche comuni sul modo di vita). Cronache Ercolanesi, Nápoles, n. 38, 2008, p. 109 160 .

- Sobre la forma de la divinidad

- PIERGIACOMI, Enrico. Mental Attraction to a Magnet-Like God. Demetrius Laco's Notion of É $\pi 1 c \pi \alpha c \mu$ óc. Cronache Ercolanesi, Nápoles, n. 45, 2015, p. 5-17.

- SANTORO, Mariacarolina. Demetrio Lacone, La forma del dio. La Scuola di Epicuro, n. 17, Nápoles: Bibliopolis, 2000. 
- Sobre la poética

- McOSKER, Michael. A New Edition of PHerc. 188 (Demetrius Laco, On Poems I). Cronache Ercolanesi, Nápoles, n. 44, 2014, p. 19-48.

d) Epicuro

- Sobre la Naturaleza

- DEL MASTRO, Gianluca. PHerc. 1416, cr 5: tre pezzi del papiro Sul tempo (PHerc. 1413). Cronache Ercolanesi, Nápoles, n. 41, 2011, p. 27-32.

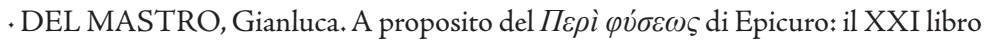
e un nuovo papiro (PHerc. 362 e 560). Lexicon Philosophicum, n. 1, 2013, p. 159-164.

- DEL MASTRO, Gianluca - LEONE, Giuliana. Addenda e subtrahenda al PHerc. 1010 (Epicuro, Sulla natura, libro II). En: DI ANTONI, Agathe. - ARRIGHETTI, Graziano. - BERTAGNA, M. Isabella. - DELATTRE, Daniel. Miscellanea Papyrologica Herculanensia. Pisa - Roma: Fabrizio Serra Editore, v. I, Biblioteca di Studi Antichi 93, 2010, p. 315-335.

- DELATTRE, Daniel. Présence ou absence d'une copie du De rerum natura à Herculanum? Réponse à Mario Capasso. En: MONET, Annick. Le Jardin romain. Épicureisme et poésie à Rome: mélanges offerts à Mayotte Bollack. Villeneuve d'Ascq: Presses de l'Université Charles de Gaulle-Lille 3, 2003, p. 109-116.

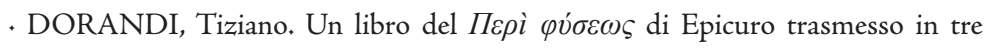
esemplari. En: ALGANZA, Minerva. - CAMACHO, José M. - FUENTES, Pedro P. Epikeia: studia graeca in memoriam J. Lens Tuero. Granada: Athos-Pérgamos, 2000, p. 103-111.

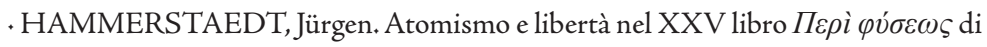
Epicuro. Cronache Ercolanesi, Nápoles, n. 33, 2003, p. 151-158.

- LEONE, Giuliana. Epicuro, Della natura, libro XXXIV (PHerc. 1431). Cronache Ercolanesi, Nápoles, n. 32, 2002, p. 7-135.

- LEONE, Giuliana. Rileggendo il XXVIII libro Della natura di Epicuro: riflessioni e proposte. Cronache Ercolanesi, Nápoles, n. 33, 2003, p. 159-164.

- LEONE, Giuliana. Per la ricostruzione dei PHerc. 1149/993 e 1010 (Epicuro, Della natura, libro II). Cronache Ercolanesi, Nápoles, n. 35, 2005, p. 15-25.

- LEONE, Giuliana. Momenti del percorso ecdotico del II libro Sulla natura di Epicuro. Cronache Ercolanesi, Nápoles, n. 36, 2006, p. 145-187.

- LEONE, Giuliana. Il secondo libro Sulla natura di Epicuro tra disegni e incisioni. Cronache Ercolanesi, Nápoles, n. 40, 2010, p. 155-172.

- LEONE, Giuliana. Sulla natura. Libro II. La Scuola di Epicuro, n. 18, Nápoles: Bibliopolis, 2012.

- LEONE, Giuliana. Eî $\delta \omega \lambda \alpha$ e nuvole: su alcune metafore e similitudini in Epicuro. Cronache Ercolanesi, Nápoles, n. 44, 2014, p. 5-18.

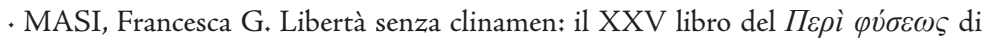
Epicuro. Cronache Ercolanesi, Nápoles, n. 36, 2006, p. 9-46. 


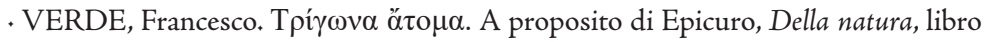
XIV col. XXXVIII 1-10 Leone. Cronache Ercolanesi, Nápoles, n. 40, 2010, p. 31-38.

e) Filodemo de Gádara

- A los amigos de la escuela

- DEL MASTRO, Gianluca. La subscriptio del PHerc. 1005 e altri titoli in caratteri distintivi nei Papiri Ercolanesi. Cronache Ercolanesi, Nápoles, n. 32, 2002, p. 245-256.

- Relación de los filósofos

* Historia de Sócrates y de su escuela

- DORANDI, Tiziano. Epicureanism and Socraticism. The Evidence on the Minor Socratics from the Herculaneum Papyri. En: ZILIOLI, Ugo. From the Socratics to the Socratic Schools. Classical Ethics, Metaphysics and Epistemology. Nueva York: Routledge, 2015, p. 168-191.

- MASSIMO GIULIANO, Fabio. PHerc. 495 - PHerc. 558 (Filodemo, Storia di Socrate e della sua scuola?). Edizione, commento, questioni compositive e attributive. Cronache Ercolanesi, Nápoles, n. 31, 2001, p. 37-79.

\section{* La Academia}

- DEL MASTRO, Gianluca. Altri frammenti dal PHerc. 1691: Filodemo, Historia Academicorum e Di III. Cronache Ercolanesi, Nápoles, n. 42, 2012, p. 277-292.

- FLEISCHER, Kilian. Der Akademiker Charmadas in Apollodors Chronik (PHerc. 1021, Kol. 31, 32). Cronache Ercolanesi, Nápoles, n. 44, 2014, p. 65-75

. FLEISCHER, Kilian. Die Schüler des Charmadas (PHerc. 1021, XXXV 32 XXXVI 14). Cronache Ercolanesi, Nápoles, n. 45, 2015, p. 49-53.

- LUPPE, Wolfgang. Zum Verkauf Platons als Sklaven in PHerc. 1021 (aus Philodems Geschichte der Akademie). Cronache Ercolanesi, Nápoles, n. 38, 2008, p. 161-163.

- SPEYER, Agustin. The earliest bust of Socrates? New observations to Philochorus in PHerc. 1021 col. 2. Cronache Ercolanesi, Nápoles, n. 31, 2001, p. 81-95.

- VERHASSELT, Gertjan. A New reading in Philodemus' Historia Academicorum (PHerc. 1021 col. 2) with observations on Dicaearchus in col. Y (F 46B Mirhady). Cronache Ercolanesi, Nápoles, n. 43, 2013, p. 17-26.

- VERHASSELT, Gertjan. Hermippus on Chaeron of Pellene (Phld., Acad. Hist., PHerc. 1021, coll. 10, 40 - 12, 4 and PHerc. 164, fr. 22 and fr. 24): edition and discussion. Cronache Ercolanesi, Nápoles, n. 45, 2015, p. 33-47.

* La escuela eleática y de Abdera / * La escuela pitagórica

- CAVALIERI, Maria C. La Rassegna dei Filosofi di Filodemo: scuola eleatica de abderita (PHerc. 327) e scuola pitagorica (PHerc. 1508)?. En: CAPASSO, Mario. Dal restauro dei materiali allo studio dei testi: aspetti della ricerca papirologica. Papyrologica Lupiensia, n. 11, Galatina: Congedo, 2002, p. 17-53. 
- ERBİ, Margherita. Il ßíos di Dionisio di Eraclea nella Stoicorum Historia di Filodemo (PHerc. 1018, coll. XXIX 5-XXXIII 4). Cronache Ercolanesi, Nápoles, $\mathrm{n}$. 43, 2013, p. 27-34.

- Sobre el buen rey según Homero

- DE SANCTIS, Dino. Omero e la sua esegesi nel De bono rege di Filodemo. Cronache Ercolanesi, Nápoles, n. 36, 2006, p. 47-64.

- DE SANCTIS, Dino. Il sovrano a banchetto: prassi del simposio e etica dell'equilibrio nel De bono rege (PHerc. 1507, coll. XVI-XXI Dorandi). Cronache Ercolanesi, Nápoles, n. 37, 2007, p. 49-65.

- DE SANCTIS, Dino. Il buon re di Filodemo tra Epicuro e Omero. Cronache Ercolanesi, Nápoles, n. 38, 2008, p. 165-177.

. FISH, Jeffrey. Philodemus' On the Good King According to Homer: Columns 21-31. Cronache Ercolanesi, Nápoles, n. 32, 2002, p. 187-232.

- FISH, Jeffrey. The Closing Columns of Philodemus' On the Good King according to Homer, PHerc. 1507, cols. 95-98 (= cols. 40-43 Dorandi). Cronache Ercolanesi, Nápoles, n. 46, 2016, p. 55-81.

- Sobre los dioses

- ESSLER, Holger. Die Arbeiten an Philodem, De dis III (PHerc. 152/157).

Der Beitrag der disegni zur die Rekonstruktion der Fragmentreihenfolge. Cronache Ercolanesi, Nápoles, n. 34, 2004, p. 153-204.

- ESSLER, Holger. Un nuovo frammento di Ermarco nel PHerc. 152/157

(Filodemo, De dis, libro III). Cronache Ercolanesi, Nápoles, n. 35, 2005, p. 53-59.

. ESSLER, Holger. Falsche Götter bei Philodem (Di III kol. 8,5 - kol. 10, 6).

Cronache Ercolanesi, Nápoles, n. 39, 2009, p. 161-205.

- ESSLER, Holger. Die Götterbewegung (Phld., Di III, kol. 10, 6 - kol.11,7). Cronache Ercolanesi, Nápoles, n. 42, 2012, p. 259-275.

. ESSLER, Holger. Freundschaft der Götter und Toten. Mit einer Neuedition von Phld., Di III, Frg. 87 und 83. Cronache Ercolanesi, Nápoles, n. 43, 2013, p. 95-112.

- ESSLER, Holger. Zum Fragment aus Chrysipps De divinatione (PHerc. 152/157, Kol. 7, 26 - Kol. 8,5). Cronache Ercolanesi, Nápoles, n. 44, 2014, p. 117-128.

. ESSLER, Holger. Ehebruch bei Göttern und Steinen (PHerc. 152/157, Frg. 78,

9). Cronache Ercolanesi, Nápoles, n. 46, 2016, p. 101-108.

- Sobre la envidia (PHerc. 1678)

- INDELLI, Giovanni. Contributi inediti di Achille Vogliano sul PHerc. 1678

(Filodemo, Opus incertum). Cronache Ercolanesi, Nápoles, n. 46, 2016, p. 109-122.

- Sobre los Estoicos

- SABATER BELTRÁ, Andrés Martín. La polémique dans la philosophie hellénistique et romaina, à la lumière du Sur les stö̈ciens (PHerc.155 et 339) de Philodème et des Vies de Diogène Laërce. En: DI ANTONI, Agathe. - ARRIGHETTI, Graziano. - BERTAGNA, M. Isabella. - DELATTRE, Daniel. Miscellanea Papyrologica Herculanensia. Pisa - Roma: Fabrizio Serra Editore, v. I, Biblioteca di Studi Antichi 93, 2010, p. 115-229. 
- Sobre la ira

- DELATTRE, Daniel. Le Sage épicurien face à la colère et à l'ivresse: une lecture renouvelée du De ira de Philodème. Cronache Ercolanesi, Nápoles, n. 39, 2009, p. 71-88.

- GIULIANO, Laura. Segni e particolarità grafiche nel PHerc. 182 (Filodemo, De ira). Cronache Ercolanesi, Nápoles, n. 35, 2005, p. 135-159.

- INDELLI, Giovanni. Filodemo e Virgilio sull'ira. Cronache Ercolanesi, Nápoles, n. 31, 2001, p. 31-35.

- INDELLI, Giovanni. Lingua e stile nell'opera Sull'ira di Filodemo (PHerc. 182). Cronache Ercolanesi, Nápoles, n. 44, 2014, p. 109-115.

- VASSALLO, Christian. Il fr. 70 Marcovich di Eraclito (= DK 22 B 85) nel De ira di Filodemo. Praesocratica Herculanensia III. Cronache Ercolanesi, Nápoles, n. 43, 2013, p. 73-94.

- Sobre los modos de vida

* Sobre la libertad de palabra

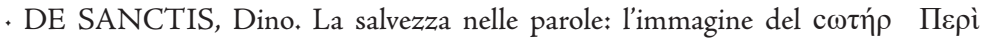
$\pi \alpha \rho \rho \eta$ cí $\alpha \varsigma$ di Filodemo. Cronache Ercolanesi, Nápoles, n. 43, 2013, p. 63-72.

- DELATTRE, Daniel. Le Franc-parler de Philodème (PHerc. 1471): reconstruction bibliologique d'ensemble du rouleau. En: DI ANTONI, Agathe. - ARRIGHETTI, Graziano. - BERTAGNA, M. Isabella. - DELATTRE, Daniel. Miscellanea Papyrologica Herculanensia. Pisa - Roma: Fabrizio Serra Editore, v. I, Biblioteca di Studi Antichi 93, 2010, p. 271-291.

- INDELLI, Giovanni - LONGO AURICCHIO, Francesca. Contributi inediti di Achille Vogliano sul PHerc. 1471 (Filodemo, La libertà di parola). Cronache Ercolanesi, Nápoles, n. 45, 2015, p. 97-145.

- INDELLI, Giovanni - LONGO AURICCHIO, Francesca. Achille Vogliano sul PHerc. 1471 (Filodemo, La libertà di parola): una postilla. Cronache Ercolanesi, Nápoles, n. 46, 2016, p. 123-125.

. WHITE, L. Michael. Ordering the Fragments of PHerc. 1471, A New Hypothesis. Cronache Ercolanesi, Nápoles, n. 39, 2009, p. 29-70.

- Sobre la muerte

- ARMSTRONG, David. All Things to All Men: Philodemus' Model of Therapy and the Audience of De Morte. En: FITZGERALD, John T. - OBBINK, Dirk. HOLLAND, Glenn S. Philodemus and The New Testament world. Leiden-Boston: Brill, 2004, p. 15-54.

- GIULIANO, Laura. Alcune considerazioni sui disegni del PHerc. 807 (opus incertum). Cronache Ercolanesi, Nápoles, n. 37, 2007, p. 93-100.

- GIULIANO, Laura. PHerc. 807: aspetti bibliologici, testo, traduzione e note. Cronache Ercolanesi, Nápoles, n. 39, 2009, p. 207-280.

- HENRY, W. Benjamin. New Light on Philodemus, On Death. Cronache Ercolanesi, Nápoles, n. 39, 2009, p. 89-102. 
. HENRY, W. Benjamin. Philodemus. On Death. Atlanta: Brill, 2010.

- Sobre la música

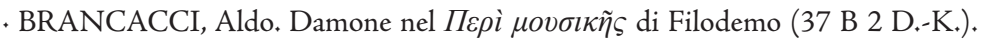
Cronache Ercolanesi, Nápoles, n. 33, 2003, p. 125-129.

- DELATTRE, Daniel. Philodème de Gadara, Commentaires sur la musique, livre IV. París: Les Belles-Lettres, 2007.

- RISPOLI, Gioia M. Teoria e storia della musica in Filodemo, Cronache Ercolanesi, Nápoles, n. 30, 2000, p. 89-102.

- RISPOLI, Gioia M. Filosofi e filosofia nel De musica di Filodemo. Cronache Ercolanesi, Nápoles, n. 33, 2003, p. 175-188.

- Sobre la piedad

- SALATI, Ornella. Mitografi e storici in Filodemo (De pietate, pars altera).

Cronache Ercolanesi, Nápoles, n. 42, 2012, p. 209-258.

- Sobre la poética

- ASMIS, Elizabeth. Philodemus on Sound and Sense in Poetry. Cronache Ercolanesi, Nápoles, n. 34, 2004, p. 5-27.

- BEER, Beate. Lukrez und Philodem: Poetische Argumenttion und poetologischer Diskurs. Basilea: Schabe, 2009.

. BOLLACK, Mayotte. L'imitation de la poésie didactique. Cronache Ercolanesi, Nápoles, n. 33, 2003, p. 199-204.

. DEL MASTRO, Gianluca. La paragraphos nei PHerc. 1425 e 1538. Cronache Ercolanesi, Nápoles, n. 31, 2001, p. 107-131.

- DEL MASTRO, Gianluca. Nuovi frammenti del II libro della Poetica di Filodemo. En: INDELLI, Giovanni - LEONE, Giuliana - LONGO AURICCHIO, Francesca. Mathesis e Mneme: Studi in memoria di Marcello Gigante. Nápoles, v. I, 2004, p. 87-94.

- IOPPOLO, Ana M. La poetica dello Stoico anonimo in Filodemo De poematis V. Cronache Ercolanesi, Nápoles, n. 33, 2003, p. 131-150.

- JANKO, Richard. Philodemus, On Poems, Book I. Oxford/New York: Oxford University Press, 2000.

- JANKO, Richard. Philodemus, On Poems, Books 3-4. Oxford/New York: Oxford University Press, 2010.

- LÓPEZ MARTÍNEZ, María Paz. La Poética de Filodemo de Gádara: estado de la cuestión. Ítaca, Barcelona, n. 19, 2003, p. 115-126.

- MACKEY, Jacob L. New Evidence for the Epicurean Theory of the Origin of Language: Philodemus, On Poems V (PHerc. 403, fr. 5, col. I). Cronache Ercolanesi, Nápoles, n. 45, 2015, p. 67-83.

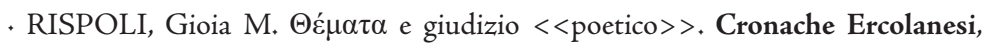
Nápoles, n. 35, 2005, p. 71-81. 
- RISPOLI, Gioia - DEL MASTRO, Gianluca. Nuove letture nel cosiddetto secondo libro della Poetica di Filodemo. En: GAGOS, Traianos. Proceedings of the Twenty-Fifth International Congress of Papyrology: Ann Arbor, July 29 - August 4, 2007. Ann Arbor: Scholarly Publishing Office, University of Michigan Library, 2010, p. 671-678.

\section{- Sobre la retórica}

- ANTONI, Agathe. Deux citations d'Euripide dans le PHerc. 1384: vers une nouvelle identification de ce livre de Philodème?. Cronache Ercolanesi, Nápoles, n. 34, 2004, p. 29-38.

- ANTONI, Agathe. Nuove letture nel PHerc. 1384 (Opus incertum). Cronache Ercolanesi, Nápoles, n. 35, 2005, p. 111-117.

- ANTONI, Agathe - DORIVAL, Gilles. Il PHerc. 1384: una nuova ipotesi di attribuzione. Cronache Ercolanesi, Nápoles, n. 37, 2007, p. 103-109.

. BLANK, David L. Aristotle's <<Academic Course on Rhetoric $>>$ and the end of Philodemus, On Rhetoric VIII. Cronache Ercolanesi, Nápoles, n. 37, 2007, p. 5-47.

. BLANK, David L. Matching Tops and Bottoms (PHerc. 1015/832). Cronache Ercolanesi, Nápoles, n. 38, 2008, p. 247-271.

- DI MATTEO, Tiziana. La retorica da Epicuro a Filodemo. Cronache Ercolanesi, Nápoles, n. 30, 2000, p. 81-88.

- DI MATTEO, Tiziana. Segni di interpunzione nel PHerc. 1669: tipologia grafica e funzione. Cronache Ercolanesi, Nápoles, n. 35, 2005, p. 119-124.

. DI MATTEO, Tiziana. Errori e correzioni nel PHerc. 1669. Cronache Ercolanesi, Nápoles, n. 36, 2006, p. 73-75.

. ERBİ, Margherita. Demostene nella Retorica di Filodemo: l'immagine del $\rho \eta ́ \tau \omega \rho$ ع̋ $\mu \pi \rho \alpha \kappa \tau o \zeta$. Cronache Ercolanesi, Nápoles, n. 38, 2008, p. 193-219.

- ERBİ, Margherita. Il retore e la città nella polemica di Filodemo verso Diogene di Babilonia (PHerc. 1004, coll. 64-70). Cronache Ercolanesi, Nápoles, n. 39, 2009, p. 119-140.

. ERBİ, Margherita. Eraclito e l'inganno della retorica in Filodemo (PHerc. 1004, coll. 57-63). Cronache Ercolanesi, Nápoles, n. 40, 2010, p. 65-74.

- ERBİ, Margherita. Il sapiente e il retore in Filodemo, Retorica VII (PHerc. 1004, col. 50). Cronache Ercolanesi, Nápoles, n. 42, 2012, p. 189-192.

- FIMIANI, Mariacristina. I papiri del IV libro della Retorica di Filodemo: segni, correzioni e caratteristiche bibliologiche (PHerc. 1423, 1673/1007 e relative scorze), Cronache Ercolanesi, Nápoles, n. 42, 2012, p. 121-188.

- FIMIANI, Mariacristina. Un proverbio nel IV libro della Retorica di Filodemo. Cronache Ercolanesi, Nápoles, n. 44, 2014, p. 77-80.

- FIMIANI, Mariacristina. Osservazioni testuali sul PHerc. 1423 (Filodemo, Retorica, libro IV, I tomo). Cronache Ercolanesi, Nápoles, n. 46, 2016, p. 95-100.

- FIORILLO, Matilde. Errori e correzioni nel PHerc. 1004 (Filodemo, Retorica VII). Cronache Ercolanesi, Nápoles, n. 43, 2013, p. 35-62. 
• FIORILLO, Matilde. I segni nel PHerc. 1004 (Filodemo, Retorica VII). Cronache Ercolanesi, Nápoles, n. 44, 2014, p. 81-107.

. LONGO AURICCHIO, Francesca. Su alcuni ä $\alpha \alpha \xi$ nella Retorica di Filodemo. Cronache Ercolanesi, Nápoles, n. 39, 2009, p. 103-106.

- NICOLARDI, Federica. Elementi per la ricostruzione del I libro del De rhetorica di Filodemo. Cronache Ercolanesi, Nápoles, n. 45, 2015, p. 55-65.

- NICOLARDI, Federica. Il successo della parola: Eschine ed Eupoli nel I libro del De rhetorica di Filodemo (Phld., Rhet. I, PHerc. 250, fr. 1 Sudhaus). Cronache Ercolanesi, Nápoles, n. 46, 2016, p. 83-93.

- Sobre la riqueza

- DEL MASTRO, Gianluca. Osservazioni sulle subscriptiones dei PHerc. 163 e 209. Cronache Ercolanesi, Nápoles, n. 33, 2003, p. 279-311.

- SCOGNAMIGLIO, Elvira. I segni nel primo libro dell'opera di Filodemo La ricchezza (PHerc. 163). Cronache Ercolanesi, Nápoles, n. 35, 2005, p. 161-181.

- SCOGNAMigliO, Elvira. Il PHerc. 163 (Filodemo, La ricchezza, I libro). Alcune osservazioni. Cronache Ercolanesi, Nápoles, n. 37, 2007, p. 85-92.

- Sobre los vicios y sus virtudes opuestas

* Sobre la adulación

- CAPASSO, Mario. Les livres sur la Flatterie dans le De vitiis de Philodème. En: AUVRAY-ASSAYAS, Clara - DELATTRE, Daniel. Cicéron et Philodème: la polémique en philosophie. París: Rue d’Ulm, 2001, p. 179-194.

* Sobre la economía

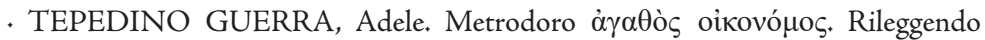
Philod., Oec. coll. XIV 23 - XV 21 (PHerc. 1424). Cronache Ercolanesi, Nápoles, n. 37, 2007, p. 67-76.

* Sobre la soberbia

- INDELLI, Giovanni. Segni, abbreviazioni e correzioni in PHerc. 1008 (Filodemo, Sui vizi, libro X). Cronache Ercolanesi, Nápoles, n. 35, 2005, p. 125-134.

. INDELLI, Giovanni. Detti e aneddoti nel PHerc. 1008 (Filodemo, I vizi, libro X). Cronache Ercolanesi, Nápoles, n. 36, 2006, p. 77-85.

- RANOCCHIA, Graziano. Aristone, Sul modo di liberare dalla superbia, nel decimo libro De vitiis di Filodemo. Florencia: Accademia La Colombaria, Serie studi, 2007.

* Sobre la insolencia

. KARAMANOLIS, George. Philodemus, Пepì ú $\rho \beta \varepsilon \omega \varsigma$ ? (PHerc. 1017). New Readings and the Philodemean Conception of Hybris. Cronache Ercolanesi, Nápoles, n. 35, 2005, p. 103-110.

\section{- Vida de Filónides}

- ASSANTE, Maria G. PHerc. 1044 (Vita Philonidis), frr. 58 e 59 Gallo. Cronache Ercolanesi, Nápoles, n. 40, 2010, p. 51-64. 
- DE SANCTIS, Dino. Il filosofo e il re: osservazioni sulla Vita Philonidis (PHerc. 1044). Cronache Ercolanesi, Nápoles, n. 39, 2009, p. 107-118.

f) Lucrecio

- Sobre la naturaleza

. ERLER, Michael. Das Bild vom <<Kind mi Menschen $>>$ bei Platon und der Adressat von Lukrez De rerum natura. Cronache Ercolanesi, Nápoles, n. 33, 2003, p. 107-116.

- KLEVE, Knut. Lucretius Herculanensis, PHerc. 395, and Disegno Oxoniense 1615. Cronache Ercolanesi, Nápoles, n. 40, 2010, p. 95-97.

\section{g) Metrodoro de Lámpsaco}

- ROSKAM, Geert. Reading fables in Epicurus' Garden. On Metrodorus, fr. 60 k. Cronache Ercolanesi, Nápoles, n. 41, 2011, p. 33-36.

. TEPEDINO GUERRA, Adele. Un frammento di Metrodoro di Lampsaco in Filodemo (PHerc. 57, col. 3). Cronache Ercolanesi, Nápoles, n. 38, 2008, p. 103-108.

- TEPEDINO GUERRA, Adele. Le opere Contro l'Eutifrone di Platone e Contro il Gorgia di Platone: per una nuova edizione dei frammenti di Metrodoro di Lampsaco. Cronache Ercolanesi, Nápoles, n. 40, 2010, p. 39-49.

- Contra los Dialécticos

- MACFARLANE, Roger T. New Readings toward Electronic Publication of PHerc. 1084. Cronache Ercolanesi, Nápoles, n. 33, 2003, p. 165-167.

\section{h) [Papiros sin identificar]}

* PHerc. 566

- DEL MASTRO, Gianluca. Il PHerc. 566: l'Echelao di Epicuro. Papyrologica Lupiensia, Lecce, n. 20-21, 2011-2012, p. 17-24.

${ }^{*}$ PHerc. 1570

- ARMSTRONG, David - PONCZOCH, Joseph A. Empedocles and Philodemus in PHerc. 1570, col. VI 9-19. Cronache Ercolanesi, Nápoles, n. 43, 2013, p. 113-116.

. PONCZOCH, Joseph A. PHerc. 1570: A Treatise on Poverty and Wealth. Cronache Ercolanesi, Nápoles, n. 39, 2009, p. 141-159.

\section{6- Filodemo y el epicureísmo}

-ARRIGHETTI, Graziano. Filodemo biografo dei filosofi e le forme dell'erudizione. Cronache Ercolanesi, Nápoles, n. 33, 2003, p. 13-30.

- ASMIS, Elisabeth. Basic Education in Epicureanism. En: LEE TOO, Yun. Education in Greek and Roman antiquity, Leiden: Brill, 2001, p. 210-239.

- ASMIS, Elisabeth. Epicurean Economics. En: FITZGERALD, John T. OBBINK, Dirk. - HOLLAND, Glenn S. Philodemus and The New Testament world. Leiden-Boston: Brill, 2004, p. 133-176. 
- CAPASSO, Mario. Who Lived in the Villa of the Papyri at Herculaneum - A Settled Question?. En: ZARMAKOUPI, Mantha. The Villa of the Papyri at Herculaneum: archaeology, reception, and digital reconstruction. Alemania: Walter de Gruyter GmbH \& Co. KG, Göttingen, 2010, p. 89-113.

- CLAY, Diskin. Philodemus on the Plain Speaking of the Other Philosophers. En: FITZGERALD, John T. - OBBINK, Dirk. - HOLLAND, Glenn S. Philodemus and The New Testament world. Leiden-Boston: Brill, 2004, p. 55-72.

- DELATTRE, Daniel. Un modèle magistral d'écriture dialectique: la Lettre à Hérodote d' Épicure. En: INDELLI, Giovanni - LEONE, Giuliana - LONGO AURICCHIO, Francesca. Mathesis e Mneme: Studi in memoria di Marcello Gigante. Nápoles, v. I, 2004, p. 149-169.

- DORANDI, Tiziano. The school and texts of Epicurus in the early centuries of the Roman empire. En: LONGO, Angela - TAORMINA, Daniela Patrizia. Plotinus and Epicurus: Matter, Perception, Pleasure. Cambridge University Press, 2016, p. 29. 50 .

- GIGANDET, Alain - MOREL, Pierre-Marie. Lire Épicure et les épicuriens. París: Presses universitaires de France, 2007.

- DORANDI, Tiziano. Diogene Laerzio, Epicuro e gli editori di Epicuro e di Diogene Laerzio. Eikasmos, Bolonia, n. 21, p. 273-301.

. DORANDI, Tiziano. <<Pratiche >> della Philosophia nella Roma repubblicana. Cronache Ercolanesi, Nápoles, n. 44, 2014, p. 167-177.

- HEBLER, Jan E. Proposte sulla data di composizione e il destinatario dell'Epistola a Meneceo. Cronache Ercolanesi, Nápoles, n. 41, 2011, p. 7-12.

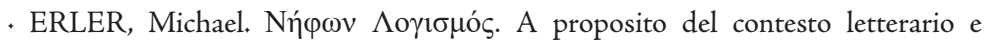
filosofico di una categoria fondamentale del pensiero epicureo. Cronache Ercolanesi, Nápoles, n. 40, 2010, p 23-29.

- ERLER, Michael. Autodidact and student: on the relationship of authority and autonomy in Epicurus and the Epicurean tradition. En: FISH, Jeffrey - SANDERS, Kirk R.. Epicurus and Epicurean Tradition. Cambridge University Press, 2011, p. 9-28.

- GIGANTE, Marcello. Virgilio all'ombra del Vesuvio. Cronache Ercolanesi, Nápoles, n. 31, 2001, p. 5-26.

- KLEVE, Knut. Epicurean Theology and Herculaneum Papyri. Cronache Ercolanesi, Nápoles, n. 33, 2003, p. 249-266.

- LONGO AURICCHIO, Francesca. Il porto della filosofia. Cronache Ercolanesi, Nápoles, n. 31, 2001, p. 27-30.

- Militello, Cesira. Filodemo storico della filosofia greca. Cronache Ercolanesi, Nápoles, n. 30, 2000, p. 103-110.

- MONET, Annick. Pratiques exégétiques au sein de l'école épicurienne: le corps et le visible. Cronache Ercolanesi, Nápoles, n. 33, 2003, p. 169-173. 
- PORTER, James I. Epicurean Attachments: Life, Pleasure, Beauty, Friendship, and Piety. Cronache Ercolanesi, Nápoles, n. 33, 2003, p. 205-227.

- SANTORO, Mariacarolina. Il pensiero teologico epicureo: Demetrio Lacone e Filodemo. Cronache Ercolanesi, Nápoles, n. 30, 2000, p. 63-70.

- SMITH, Martin Ferguson. Herculaneum and Oinoanda, Philodemus and Diogenes: Comparison of Two Epicurean Discoveries and Two Epicurean Teachers. Cronache Ercolanesi, Nápoles, n. 33, 2003, p. 267-278.

- TEPEDINO GUERRA, Adele. La Scuola di Epicuro: Metrodoro, Polieno, Ermarco. Cronache Ercolanesi, Nápoles, n. 30, 2000, p. 35-44.

\section{7- Manuales}

- AUVRAY-ASSAYAS, Clara - DELATTRE, Daniel. Cicéron et Philodème: la polémique en philosophie. París: Rue d'Ulm, 2001.

- DELATTRE, Daniel. La Villa des Papyrus et les rouleaux d'Herculanum. La Bibliothèque de Philodème. Lieja (Bélgica): Éditions de l'Université de Liège, Cahiers du CeDoPaL, n. 4, 2006.

- DELATTRE, Daniel - PIGEAUD, Jackie. Les Épicuriens. París: Gallimard, 2010 .

- GALLO, Italo. Studi di papirologia ercolanese. Nápoles: M. D’ Auria, 2002.

- SIDER, David. The Library of the Villa dei Papiri at Herculaneum. Los Ángeles: Getty Publications, 2005.

- SWAIN, Simon. Economy, Family and Society from Rome to Islam: A critical Edition, English Translation, and Study of Bryson's Management of the Estate, Cambridge University Press, 2013.

. TSOUNA, Voula. The Ethics of Philodemus. Oxford: Oxford University Press, 2006.

\section{5+ Conclusiones}

El mensaje de Epicuro es antiguo y moderno. Propone valores eternos, que se mantienen vigentes en la actualidad: salud, tranquilidad de espíritu, amistad, tolerancia y felicidad. Estamos convencidos de que no sólo los científicos y académicos sino también, y gracias a ellos, el conjunto de la sociedad se beneficiaría de un conocimiento más profundo de una comunidad que vivió practicando el hedonismo con tanta intensidad como el pragmatismo. Filodemo, Diógenes de Enoanda y otros representantes de esta corriente de pensamiento -marginal en su momento- plantearon unas soluciones para hacer frente a los problemas del individuo y de la sociedad que fueron originales y distintas a las que proponían otras escuelas que, por entonces, gozaron de mayor éxito, como fueron el platonismo y/o el estoicismo y fueron 
estas últimas, precisamente, las que acabaron imponiéndose como ortodoxia educativa en occidente.

Una perspectiva especialmente sugerente desde la que abordar el epicureísmo antiguo es la de género. A diferencia de lo que era frecuente en otras escuelas de filosofía, en las comunidades epicúreas, está perfectamente atestiguada la presencia de mujeres desde su fundación así como lo está el activo papel que éstas desempeñaron en la intensa vida del Jardín: no se limitaban a ser compañeras de filósofos sino que expresamente se recoge su participación en todas las actividades de la escuela.

Esa originalidad del Jardín se plasma paradójicamente también en la medida en la que el cristianismo se nutrió también del mensaje de la escuela, basta recordar el uso que hace Pablo de Tarso del valor terapéutico de la libertad de palabra epicúrea durante el periodo de fundación de las primeras comunidades paulinas.

Dos de los desafíos que han de afrontar los expertos en los materiales encontrados en la ciudad antigua de Herculano son, por un lado, divulgar el conocimiento de esta rica y compleja filosofía y, por otro, demostrar su utilidad pedagógica, con el fin de que, en un futuro próximo, dicho conocimiento pueda ayudar a educadores, científicos, médicos y/o psicólogos en la tarea de construir una sociedad más pacífica y tolerante, formada por individuos responsables, felices e inteligentes en el manejo de sus emociones.

En un momento en el que disciplinas como la filología, la historia y la filosofía están en crisis e incluso se cuestiona su papel en los planes de estudio -reducción de la carga lectiva en secundaria, mientras en prensa se plantea la conveniencia de introducir estas materias en primaria $y$, por ejemplo, la ONU pide al gobierno español que se recupere la asignatura de Educación para la Ciudadanía [http://www.elmundo.es/ espana/2015/07/08/559c40ec268e3ef81b8b4589.html -fecha de la consulta: 1-V-2017] - se hace más necesario que nunca demostrar la utilidad de la misma mediante proyectos como el que presentamos.

Nuestro horizonte como humanistas debería proyectarse también a largo plazo para poner al alcance de la sociedad los instrumentos de estudio adecuados para valorar, en la medida que le corresponde -sin duda, mayor que la que le ha correspondido hasta ahora-, la contribución al humanismo de la escuela de Epicuro. Así como Freud aplicó lo que había aprendido de sus lecturas sobre tragedia griega, estamos convencidos de que llegará un día en que expertos de otras disciplinas entenderán también el potencial educativo y la utilidad práctica de la lectura de los tratados epicúreos. De hecho, autores 
como Marinoff - Más Platón y menos Prozac-o Damásio -El error de Descartes: la emoción, la razón y el cerebro bumano y En busca de Spinoza: Neurobiología de la emoción y los sentimientos-, entre otros, ya lo han entendido. Cuando llegue ese momento, nos gustaría estar en condiciones de afirmar que nuestro esfuerzo como filólogos, académicos y/o humanistas ha contribuido a hacerlo posible.

Debemos asumir el estimulante desafío que comporta a los investigadores de las disciplinas referidas anteriormente -Filología Clásica, Arqueología, Historia Antigua y Filosofía- poner al alcance de cualquier persona culta con inquietudes humanísticas todo este rico y complejo legado.

Por todo ello, el mensaje de alegría de vivir, serenidad y tolerancia de Epicuro está más vivo y, si cabe, es hoy más necesaria que nunca la colaboración, en la medida de nuestras posibilidades, entre los departamentos e instituciones de diferentes universidades europeas, americanas y de todos aquellos ámbitos que desarrollan activamente actividades relacionadas con el recinto arqueológico de Herculano con el objetivo de saldar la deuda cultural de Occidente con respecto a una de las corrientes filosóficas más injustamente criticada (e incluso calumniada) desde la Antigüedad.

\begin{abstract}
Our purpose is to assess the contribution to the Humanism of the legacy hidden for twenty centuries and appeared in one of the most interesting archaeological sites that we know now, the Villa dei Papiri at Herculaneum, buried by lava from Vesuvius in 79 AD. Since 1750, it began to be excavated and it has allowed us to discover a luxurious mansion, great value articles and the only library that has come down to us from Antiquity. This content is made up by almost two thousand rolls of papyrus written in Greek and attributed to an author born in Gadara -Syria- called Philodemus. Twenty centuries ago it brought together the followers of the school, whose main objective was the pursuit of happiness. We are convinced that the message of serenity, zest for life and tolerance of Epicurus is more alive than ever. Our aim is to make available to a great number of people, not just to those interested in Philosophy, Literature, History and Archeology of Hellenistic and Imperial Periods, but also any educated person with humanistic concerns this rich and complex legacy.
\end{abstract}

Key words: Herculaneum, Villa dei Papyri, Philodemus, bibliography update, XXI Century. 